\title{
Blockchain no setor público: uma revisão sistemática de literatura
}

\section{Blockchain in public sector: a systematic literature review}

\author{
Euber Chaia Cotta e Silva ${ }^{1}$, Rodrigo Moreno Marques ${ }^{2}$ \\ ${ }^{1}$ Universidade FUMEC, Belo Horizonte, Minas Gerais, Brasil. ORCID: https://orcid.org/0000-0003-0166-4303 \\ 2 Universidade Federal de Minas Gerais (UFMG), Belo Horizonte, Minas Gerais, Brasil. ORCID: https://orcid.org/0000-0002-6320-4874
}

Autor para correspondência/Mail to: Euber Chaia Cotta e Silva, euberchaia@gmail.com

Recebido/Submitted: 08 de março de 2021; Aceito/Approved: 18 de junho de 2021

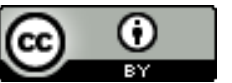

Copyright (c) 2021 Silva \& Marques. Todo o conteúdo da Revista (incluindo-se instruções, política editorial e modelos) está sob uma licença Creative Commons Atribuição 4.0 Internacional. Ao serem publicados por esta Revista, os artigos são de livre uso em ambientes educacionais, de pesquisa e não comerciais, com atribuição de autoria obrigatória. Mais informações em http://revistas.ufpr.br/atoz/about/submissions\#copyrightNotice.

\begin{abstract}
Resumo
Introdução: por meio de portais governamentais, é possível aumentar a transparência e a participação da sociedade no governo. A tecnologia blockchain tem sido apontada como uma alternativa para aumentar a transparência governamental e reduzir custos no setor público. Nesse contexto, o artigo teve como objetivo responder à questão principal: qual o atual panorama das aplicações da tecnologia blockchain no setor público? Método: foi feita uma revisão sistemática de literatura por meio do indexador Google Acadêmico, tendo como recorte temporal os trabalhos publicados entre 2008 e 2018. Foram utilizadas as seguintes palavras-chave: "government transparency", "open government", "e-government", "blockchain applications", "blockchain application", "transparência governamental", "governo aberto", "setor público", "governo eletrônico", "aplicações blockchain" e "aplicação blockchain". Resultados: foram detectadas diversas áreas consideradas de interesse do setor público para implantações da tecnologia blockchain, bem como áreas onde ela já se encontra em uso. Conclusões: o tema tem ganhado destaque em diversas áreas, como pode ser comprovado por meio desta pesquisa, que encontrou relatos de uso nos seguintes setores: energia elétrica, imobiliário, medicina, gerenciamento de identidades, agricultura, transações comerciais, mercado financeiro, tributação, cidades inteligentes, contratos inteligentes, administração governamental e votação eletrônica, entre outros. Esta revisão sistemática de literatura indica que a tecnologia em tela tem potencial para ajudar o setor público.
\end{abstract}

Palavras-chave: Blockchain; Cadeia de blocos; Setor público; Governo; Transparência governamental.

\begin{abstract}
Introduction: through government portals, it is possible to increase transparency and the participation of society in government. Blockchain technology has been touted as an alternative to increase government transparency and reduce costs in the public sector. In this context, the article aimed to answer the main question: what is the current panorama of blockchain technology applications in the public sector? Method: it carries out a systematic literature review using the Google Scholar index, with a frame of works published between 2008 and 2018. The following keywords were used: "government transparency", "open government", "e-government","blockchain applications", "blockchain application", "government transparency", "open government", "public sector", "e-government", "blockchain applications" and "blockchain application". Results: it detects several areas of interest to the public sector for blockchain technology deployments, as well as areas where it is already in use. Conclusions: the theme has gained prominence in several areas, as can be seen through this research, which found reports of use in the following sectors: electricity, real estate, medicine, identity management, agriculture, commercial transactions, financial market, taxation, cities smartphones, smart contracts, government administration, and electronic voting, among others. This systematic literature review indicates that the technology on screen has the potential to help the public sector.
\end{abstract}

Keywords: Blockchain; Block chain; Public sector; Government; Government transparency.

\section{INTRODUÇÃO}

As tecnologias da informação e comunicação são cada vez mais adotadas no setor público. Por meio de portais governamentais, é possível aumentar a transparência das instituições públicas e fomentar a participação da sociedade no governo. Uma das premissas fundamentais da democracia é tornar visíveis as ações do governo (Pinho, 2008; Prado \& Loureiro, 2006).

Conforme Barbosa (2017, p. 88-89), há uma evolução de uma hierarquia arquivística para uma estrutura de rede no setor público. As tecnologias em uso hoje criam novas formas de os cidadãos expressarem suas opiniões e coordenarem esforços. Uma destas tecnologias é a blockchain, e sua aplicação no governo permite, por exemplo, o registro de transações administrativas como certidões de nascimento e registro de propriedades. Nesse sentido, a tecnologia blockchain tem potencial para ser um importante instrumento para transparência governamental. De acordo com Berryhill, Bourgery, e Hanson (2018, p. 10), a tecnologia blockchain pode ser definida como um "sistema digital de contabilidade distribuída que funciona como um registro aberto, compartilhado e confiável que realiza transações entre as partes e não é armazenado por uma autoridade central". A tecnologia blockchain pode se tornar um importante instrumento para o setor público, se empregada para tornar os processos públicos mais transparentes, seguros e confiáveis (Welzel, 20017). 
Porém, não obstante essas vantagens apontadas pela literatura acadêmica, Ferreira, Pinto, e Santos (2017) apresentam diversos problemas da blockchain: baixo nível de amadurecimento da tecnologia, poucas transações por segundo, alta latência (tempo para processamento de informações), desperdício de recursos (gasto de poder de processamento e energia altos), baixa usabilidade, possibilidade de forjar transações ao controlar mais de $50 \%$ da rede.

Diante desse contexto, o artigo aborda o uso da tecnologia blockchain em aplicações para transparência governamental, partindo da pergunta: qual o atual panorama das aplicações da tecnologia blockchain no setor público? Considerando esta questão, o objetivo do artigo é apresentar as áreas nas quais a tecnologia blockchain está em uso ou tem potencial para ser usada pelo setor público. Para atingir tal objetivo, foi feita uma Revisão Sistemática de Literatura (RSL) que tomou como corpus os artigos indexados pelo Google Acadêmico.

O artigo está estruturado em quatro seções. Após a introdução, que aborda a transparência governamental, trazendo os seus principais conceitos, na visão de diversos autores e também apresenta a tecnologia blockchain, com uma visão sobre seu funcionamento, estrutura e vantagens. A seção dois traz a metodologia adotada na RSL. Na sequência, são apresentados os resultados da RSL, por meio da qual são respondidas as perguntas de pesquisa deste trabalho e, por último, é apresentada a conclusão do trabalho.

\section{Transparência Governamental}

O objetivo da transparência governamental deve ser prover acesso pelos cidadãos às informações governamentais, tornando mais democráticas e estreitas as relações entre Estado e sociedade. Tentar elevar o nível de transparência pública é um dos desafios da administração pública (Madrigal, 2016). É importante a divulgação efetiva de todas as ações governamentais, que não sejam sigilosas, para fortalecer a democracia.

O envolvimento de diferentes classes sociais no acompanhamento da gestão deve ser auxiliado pelo aumento da transparência. Quando a divulgação de informações fica restrita a alguns grupos, a promoção da democracia é inibida, ferindo os princípios constitucionais da legalidade, impessoalidade, moralidade e eficiência (Figueiredo \& Santos, 2014).

Para Jardim (1995), a noção de transparência administrativa é um dos requisitos de controle da sociedade sobre o Estado. A separação das esferas política e administrativa torna-se incompatível com a administração pública moderna. Por isso, o rígido controle dos cidadãos sobre o Estado e do próprio Estado sobre si mesmo se torna imprescindível.

\section{A Tecnologia Blockchain}

Para Šurda (2012), blockchain é um "livro" distribuído, e os computadores conectados diretamente à Internet podem ter uma cópia completa dele. Os blocos (conjunto das transações dos últimos 10 minutos com data e hora de realização, além de um código que identifica a sequência de blocos anteriores) são ordenados sequencialmente, formando o ledger (livro-razão). A sequência correta e a integridade dos dados são confirmadas por criptografia. Segundo Foroglou e Tsilidou (2015) esse livro público está em constante crescimento, na medida em que os novos blocos "concluídos" lhe são adicionados. O bloco mais recente é a parte atual de uma blockchain, que registra as transações recentes e essas, uma vez concluídas, são registradas na ledger. Quando esse bloco atual é concluído, um novo bloco é gerado com o registro das novas transações e ambos são ligados em ordem cronológica linear, com cada um contendo um hash do anterior. Cada bloco tem um tamanho variável, dependendo do tipo da blockchain em uso, assim como sua estrutura, havendo, geralmente, alguns atributos essenciais, como o cabeçalho do bloco, ponteiros para blocos anteriores, data e hora, contador de transações e transações (Bashir, 2017).

Uma característica importante da blockchain é que os registros a ela adicionados são praticamente imutáveis. Apesar da possibilidade de reverter as alterações, executar uma alteração é quase impossível por exigir uma quantidade inacessível de recursos de computação (Bashir, 2017). Outra importante característica da blockchain é que sua estrutura permite que diferentes organismos realizem transações entre si em uma rede de computadores na qual ninguém é validado por uma instituição central (Horiuchi, 2018).

Após essas breves considerações teóricas, na seção seguinte são apresentados os procedimentos da Revisão Sistemática de Literatura (RSL) realizada.

\section{METODOLOGIA}

Foram feitas pesquisas no indexador Google Acadêmico, com filtro em trabalhos publicados entre 2008 e 2018. Justifica-se o ano inicial por ser quando a tecnologia blockchain começou a aparecer no artigo de Horiuchi (2008). Foram utilizadas as seguintes palavras-chave: "government transparency", "open government", "e-government", "blockchain applications", "blockchain application", "transparência governamental", "governo aberto", "setor público", "governo eletrônico", "aplicações blockchain" e "aplicação blockchain". 
Quanto à escolha do indexador de bases de dados, optou-se por usar o Google Acadêmico, visando a um maior alcance de artigos e pela opção de empregar uma base aberta (open access) para um melhor aproveitamento dos resultados da RSL por outros pesquisadores. Para a busca, foram utilizadas as palavras-chaves anteriormente escolhidas, com ocorrência em qualquer lugar do artigo, incluindo patentes e citações

As palavras-chaves foram combinadas em dois grupos em razão de uma limitação máxima de caracteres do indexador: Grupo 1: ("government transparency"OR "open government"OR "e-government") AND ("blockchain applications"OR "blockchain application") e grupo 2: ("transparência governamental"OR "governo aberto"OR "setor público"OR "governo eletrônico") AND ("aplicações blockchain"OR "aplicação blockchain").

O resultado das buscas foi de 162 resultados para o grupo 1, com termos em inglês e quatro para o grupo 2 , com os termos em português. Foi feita uma separação das principais publicações sobre o tema de acordo com os termos anteriormente citados. Após esse processo, foi feita uma filtragem da relevância do artigo, por meio de uma leitura que buscou nos textos indícios ou pretensão de uso da tecnologia blockchain no setor público. Primeiramente, foi feita leitura dos resumos e, após, seleção final via leitura completa. Mais informações dos critérios adotados para seleção na subseção "Critérios de seleção de artigos".

\section{Questão norteadora da RSL}

As revisões de literatura fazem parte de qualquer projeto de pesquisa. Nestas revisões, o pesquisador avalia o território intelectual para especificar uma lacuna de pesquisa. No entanto, as revisões tradicionais não têm um protocolo adequado, podendo ser influenciadas pelo pesquisador do qual pode fugir uma massa de evidências contraditórias. Por isso, revisões sistemáticas aparecem como uma solução, pois expõem os estudos a um rigoroso escrutínio metodológico de forma a reduzir o erro humano e o preconceito (Tranfield, Denyer, \& Smart, 2003).

O objetivo da RSL foi fazer um levantamento bibliográfico para mapear o uso da tecnologia blockchain no setor público e identificar quais as áreas de uso e tipos de aplicações voltadas para a transparência governamental. A questão principal $[\mathrm{QP}]$ que norteou a RSL foi: Qual é o atual panorama das aplicações da tecnologia blockchain no setor público?

Para auxiliar na resposta da questão principal, foram criadas duas questões específicas [QE]:

[QE1] Para quais áreas e que tipos de aplicações da tecnologia blockchain estão sendo propostas, segundo a literatura acadêmica? [QE2] Que tipos de aplicações da tecnologia blockchain estão voltados para transparência governamental, de acordo com a literatura acadêmica?

\section{Critérios de Seleção de Artigos}

Os critérios para exclusão utilizados seguiram cinco fases: Fase 1 - Exclusão por forma de acesso; Fase 2 Exclusão por citações; Fase 3 - Exclusão por idioma; Fase 4 - Exclusão por resumo e/ou palavras-chave; Fase 5 - Exclusão por leitura completa.

$\mathrm{Na}$ primeira fase, apenas artigos abertos (open access) foram aceitos e com os formatos: pdf, doc ou html. Na segunda fase, artigos sem no mínimo uma citação foram cortados. Na terceira fase, foram selecionados apenas os artigos em inglês ou português. Na quarta fase, a exclusão foi feita após a leitura do resumo e palavras-chave. Os artigos cujo resumo se mostrava fora do objetivo proposto foram excluídos. Por último, na quinta etapa, foi feita a leitura completa dos artigos restantes e definidos critérios específicos para exclusão, de acordo com objetivo da pesquisa. Foram definidas pontuações para cada um dos critérios e a avaliação. Artigos com uma pontuação final (somatório) inferior a sete foram excluídos. Como resumo para entender a seleção é possível verificar que caso o uso ou proposta de uso da tecnologia blockchain não se confirmasse para o setor público, o artigo era excluído. As questões de pesquisa utilizadas, bem como suas respectivas notas podem ser vistas na Tabela 1.

\begin{tabular}{lc}
\hline Questões de Pesquisa & Pontuação \\
\hline Atende ao objeto da pesquisa? & 1 \\
Há resposta aos objetivos da pesquisa proposta? & 1 \\
Há menções sobre o uso da tecnologia blockchain no setor público? & 4 \\
É possível traçar um panorama do uso do blockchain no setor público? & 4 \\
Total & 10 \\
\hline
\end{tabular}

Tabela 1. Critérios de exclusão - Fase 5, leitura completa.

Source: Elaborado pelos autores (2021).

Foram encontrados inicialmente cento e sessenta e seis artigos. A Tabela 2 demonstra quantos foram selecionados após cada fase de exclusão: 


\begin{tabular}{lc}
\hline Fase & Quantidade de Artigos \\
\hline 1 - Forma de acesso & 136 \\
2 - Citações & 82 \\
3 - Idioma & 78 \\
4 - Resumo e/ou palavras-chave & 55 \\
5 - Leitura completa & 39 \\
\hline
\end{tabular}

Tabela 2. Quantidade de artigos selecionados após cada fase de exclusão. Fonte: Elaborado pelos autores (2021).

A filtragem a cada fase ajudou a diminuir o escopo para uma leitura mais minuciosa, que visava à verificação se o texto realmente atendia ao critério proposto no artigo, tendo a próxima seção tratado de mostrar esta análise.

\section{Artigos Selecionados}

Dos 166 artigos da pesquisa inicial no Google Acadêmico, foram selecionados 39 que atenderam aos critérios estabelecidos. Os títulos, os nomes dos(as) autores(as), as datas e áreas de publicação dos trabalhos encontrados estão na tabela ??.

\begin{tabular}{l} 
Título dos Artigos \\
\hline Roadblocks to application of blockchain in in- \\
dian agriculture - a study on madhya pradesh \\
Does Technology Against Corruption Always \\
Lead to Benefit? The Potential Risks and Chal- \\
lenges of the Blockchain Technology \\
Are emerging technologies helping win the \\
fight against corruption in developing coun- \\
tries?
\end{tabular}

Don't be fooled by the blocks that it got

A Blockchain Technology Evolution between Business Process Management (BPM) and Internet-of-Things (loT)

Semantic-enhanced blockchain technology for smart cities and communities

Upgrading blockchains: Smart contract use cases in industry

Blockchain 2.0 - From Bitcoin Transactions to Smart Contract applications

Cryptolaw for Distributed Ledger Technologies: A Jurisprudential Framework

Blockchain-enabled self-sovereign identity

The Application of Digital Identity in the United States

Changing Governance Models by Applying Blockchain Computing

Blockchain from Public Administration Perspective: Case of Estonia

Unlocking Blockchain: Embracing New Technologies to drive Efficiency and Empower the Citizen

Blockchain technology: A general purpose technology for the decentralization of governance? Blockchain Technologies as A Stimulator of Institutional Transformations of the World Financial System

Enforcing Constitutional Rights Through Computer Code

Blockchain - Attack on and Chance for the Public Sector

Blockchain: Perspectives on Research, Technology and Policy

\begin{tabular}{cc} 
Autores(as)/ Ano & Áreas de Aplicação \\
\hline Dubey (2018) & Agricultura \\
Kim e Kang (2017) & Anticorrupção
\end{tabular}

Adam e Fazekas (2017)

Anticorrupção

van Nes (2017)

Hussein, Hamed, e Eldeen (2017)

Ruta et al. (2016)

Ream, Chu, e Schatsky (2016)

Kehrli (2016)

Reyes (2018)

van Wingerde (2017)

Duffy, Goudovitch, e Fedorov (2016)

Young (2018)

Jalakas (2018)

Cadeia de suprimentos

Cidades

inteligentes

Cidades inteligentes

Contratos

inteligentes

Contratos

inteligentes

Emissão de ações societárias

Gerenciamento de identidades

Gerenciamento de identidades

Governo / Cidadão

Governo / Cidadão

Hughes, Graham, Rowley, e Lowe (2018)

Flament (2015)

Governo / Cidadão

Kussy, Pobirchenko, Shutaieva, e Kakutich (2018)

Young (2017)

Governo / Cidadão

Welzel (20017)

Governo / Cidadão

Governo / Cidadão 


\begin{tabular}{l} 
Título dos Artigos \\
\hline Blockchain Technology for Recordkeeping Le- \\
mieux, Hofman, Batista, e Joo (2019) \\
FHIRChain: Applying Blockchain to Securely \\
and Scalably Share Clinical Data \\
Pattern Based Evaluation of Blockchain Tech- \\
nology as a Catalyst for Business Model Innova- \\
tion: Exploratory Research with Focus on the \\
Potential Implications for e-Health \\
Blockchain and Financial Market Innovation
\end{tabular}

Risks And Opportunities For Systems Using Blockchain And Smart Contracts

Digitalization of Land Records: From Paper to Blockchain

Blockchain - can this new technology really revolutionize the land registry system?

Tendências para sistemas microgrids em cidades inteligentes: uma visão sobre a blockchain Applying Blockchain Technology to Electric Power Systems

Scaling blockchain for the energy sector

Attention to Disruption and Blockchain Creates a Viable Real Estate Economy

Blockchain Based E-Stamp Procurement System with Efficient Consensus Mechanism and Fast Parallel Search

A Survey of Blockchain Technologies for Open Innovation

Closing in on the Holy Grail of World Trade: Using Blockchain to Expand Southeast Asia's Trade

Exploring the Future of Taxation: A Blockchain Scenario Study

Blockchain: Foundational Technology To Change The World

Beyond bitcoin: Blockchain is coming to disrupt your industry

Blockchain 3.0: Towards a Secure Ballotcoin Democracy through a Digitized Public Ledger in Developing Countries

Crypto-voting, a Blockchain based e-Voting System

Blockchains and Voting: Somewhere between hype and a panacea
Autores(as) / Ano

Áreas de Aplicação

Manutenção de registros

Zhang, White, Schmidt, Lenz, e

Rosenbloom (2018)

Šalehar (2017)

Medicina

Medicina

Lewis, McPartland, e Ranjan (2017)

Staples et al. (2017)

Benbunan-Fich e Castellanos (2018)

Barbieri e Gassen (2017)

Gabrich, Coelho, e Coelho (2017)

Livingston, Sivaram, Freeman, e Fiege (2018)

Dahlquist \& Hagström (2017)

Veuger (2017)

Singh e Vardhan (2018)

De La Rosa et al. (2017)

Suominen (2018)

van Rijswijk, Hermsen, e Arendsen (2019)

Khudnev (2017)

Schatsky e Muraskin (2015)

Dogo, Nwulu, Olaniyi, Aigbavboa, Votação eletrônica e Nkonyana (2018)

Fusco, Lunesu, Pani, e Pinna (2018)

Nasser, Okoye, Clark, e Ryan Votação eletrônica (2018)
Mercado financeiro

Pagamentos de remessas; Registros

de dados abertos;

Cadeia de

suprimentos agrícola

Registro de bens imóveis

Registro de bens imóveis

Setor de energia elétrica

Setor de energia elétrica

Setor de energia elétrica

Setor imobiliário

Setor imobiliário

(selos eletrônicos)

Survey por diversas áreas

Transações comerciais

Tributação

Vários usos

Vários usos

Votação eletrônica

Tabela 3. Artigos selecionados.

Fonte: Elaborado pelos autores (2021).

Após a leitura completa dos textos, todos os artigos que não ajudavam a traçar um panorama de uso da blockchain no setor público foram excluídos, pois o foco desta RSL é proceder a um levantamento do atual cenário da tecnologia blockchain no setor público.

\section{DISCUSSÃO DOS RESULTADOS DA RSL}

Nesta seção são apresentados e discutidos os resultados da RSL feita. Através dos artigos selecionados, foi possível responder as perguntas a seguir. 
[QE1] Para quais áreas e que tipos de aplicações a tecnologia blockchain está sendo proposta, segundo a literatura acadêmica?

a) Setor de energia elétrica:

Gabrich et al. (2017) afirmam que uma área que poderia se beneficiar da tecnologia blockchain é o setor de energia elétrica pelo uso da energia excedente oriunda da geração distribuída. A blockchain entraria, nesse caso, na etapa de armazenamento de dados, que poderia ser feita de forma confiável na compra, venda ou doação de energia. Já Livingston et al. (2018), fizeram entrevistas com startups que desejavam criar novos mercados ou aprimorar os existentes para o comércio de eletricidade, facilitando transações ponto a ponto, facilitando o pagamento de cobranças de consumo de veículos elétricos, rastreando a produção ou arrecadando fundos para energia limpa. Para Dahlquist e Hagström (2017), a tecnologia blockchain é uma alternativa para adoção no setor de energia elétrica. O foco do trabalho é um estudo de caso de um sistema de pagamento para estradas eletrificadas, com tecnologia para transferência de energia para veículos.

b)Setor imobiliário ou registro de bens imóveis:

Veuger (2017) traz uma proposta voltada ao setor imobiliário, por meio da qual se poderia fazer um cadastro com registro de propriedade usando blockchain, que pode ser uma forma mais eficiente de fazer o registro para a transferência de propriedades. O autor destaca que deve haver uma conexão clara entre a administração na blockchain e a propriedade física. Outros autores que discutem as vantagens do uso da blockchain nesse mesmo setor são Singh e Vardhan (2018) (2018), que focam no problema de transferência de bens imóveis na Índia. O trabalho de Benbunan-Fich e Castellanos (2018) destaca essa área com um comparativo do registro de terras na Geórgia e Honduras. A adesão da Georgia a blockchain seria mais fácil, pois os registros estão digitalizados, já Honduras teria a tarefa adicional de digitalização. Lemieux et al. (2019) fizeram um estudo sobre os usos potenciais da tecnologia blockchain para manutenção de registros. Na área financeira, sistemas de pagamento, emissão de ações corporativas e liquidação de tesouraria poderiam ocorrer no mesmo dia; fora da área financeira, o uso em contratos e testamentos inteligentes, proteção de informações pessoais identificáveis na blockchain em data lake e automação de procedimentos de back office.

c) Medicina:

Na área da medicina, o artigo de Zhang et al. (2018) descreveu um protótipo, chamado de FHIRChain, projetado pelos autores para fornecer aos pacientes um suporte mais colaborativo à decisão clínica, usando a tecnologia blockchain e os recursos rápidos de interoperabilidade em assistência médica. Outro autor que faz uma análise do uso da blockchain no campo da medicina é Šalehar (2017), que aborda os possíveis desenvolvimentos no mercado e-Health. Alguns exemplos são o conceito de plataforma para tratamento personalizado, com coleta de dados do paciente e conexão de diferentes prestadores de cuidados, como de voluntários a pacientes idosos.

d) Gerenciamento de identidades:

O artigo de van Wingerde (2017) propõe um novo paradigma, o gerenciamento de identidade autossoberano, que seria possível por meio da tecnologia da blockchain. A proposta de um sistema descentralizado, de forma que o próprio sujeito consiga fazer a administração dos seus dados, é apresentada como uma instigante solução. Duffy et al. (2016) trazem um interessante estudo sobre o uso da blockchain para gerenciamento de identidades. Os autores dizem que, para tal, há características atraentes da tecnologia, como ser descentralizada e distribuída, o que poderia ajudar a reduzir fraudes e cumprir as regulamentações existentes. Entretanto, conforme alegam os autores, essa ainda não é uma solução viável para um sistema de gerenciamento de identidade em nível nacional em um país que não seja totalmente digital.

e) Agricultura:

Na tese de Dubey (2018), é citado o projeto IndiaChain do governo indiano, cujo objetivo é criar uma rede blockchain no país. Algumas áreas do NITI Aayog (principal banco de ideias do governo da Índia) preveem os seguintes usos de blockchain: digitalização de certificados, educação, criação de registro de terras, distribuição de eletricidade e agricultura. Esta última área é o foco do trabalho do autor, que alega que, como o setor emprega mais da metade da população indiana, a proposta traria bons impactos sociais, econômicos e políticos.

f) Transações Comerciais:

O artigo de Suominen (2018) discute como a blockchain está melhorando diversas áreas do sudeste asiático, entre as quais o gerenciamento da cadeia de suprimentos, a logística transfronteiriça, os procedimentos alfandegários e o financiamento comercial. De acordo com o autor, empresas e governos regionais já são líderes mundiais no uso da tecnologia blockchain para tornar mais eficiente a movimentação de mercadorias entre países, melhorando a logística, o financiamento comercial, o desembaraço aduaneiro e a rastreabilidade da cadeia de suprimentos.

g) Mercado Financeiro:

Lewis et al. (2017) argumentam que a tecnologia blockchain provavelmente será fonte fundamental de inovação futura no mercado financeiro por permitir a criação de registros imutáveis de transações acessíveis por todos os participantes de uma rede. Apesar de todas as aplicações da tecnologia blockchain estarem em estágios iniciais de desenvolvimento, os autores defendem que há muitas aplicações promissoras nos mercados financeiros, pois elas têm o potencial de fornecer grandes ganhos de eficiência em negócios, que, atualmente, exigem intermediação 
dispendiosa.

h) Tributação:

No trabalho de van Rijswijk et al. (2019), é apresentado um instrumento voltado para o planejamento exploratório de cenários, com o objetivo de facilitar a tomada de decisões estratégicas. Os autores aplicaram o método de planejamento de cenário exploratório na tecnologia blockchain e exploraram as diferentes maneiras pelas quais os desenvolvimentos tecnológicos em relação à blockchain poderão impactar a tributação em 2025. Os pesquisadores analisam os cenários apresentados, citando, por exemplo, que as autoridades não devem superestimar a influência da tecnologia blockchain no tipo de inovações emergentes. Alegam também que, por serem ainda imaturas, elas podem ter impacto negativo na sua aderência a desenvolvimentos futuros.

i) Cidades Inteligentes:

O artigo de Hussein et al. (2017) traz um estudo abrangente da tecnologia blockchain, examinando os esforços de pesquisa dessa tecnologia. Foi apresentando um ciclo de vida proposto para a blockchain, visando à melhoria do gerenciamento de processos de negócios (BPM) e aos conceitos de Internet das Coisas (IoT). Os autores realizaram uma prova prática dessa relação para uma cidade inteligente. Ruta et al. (2016) propõem uma nova Arquitetura Orientada a Serviços (SOA), com base numa blockchain semântica. Para os autores, a tecnologia blockchain pode substituir sistemas transacionais de larga escala que ainda sofrem com estratégias de gerenciamento de confiança não viáveis. Há diversas áreas de interesse para cidades inteligentes citadas no trabalho, como mercados de recursos materiais e imateriais e colaboração entre entidades autônomas.

j) Contratos Inteligentes:

Os contratos inteligentes parecem ser uma solução viável para a substituição de contratos de papel ou documentos digitais, segundo Ream et al. (2016). Esses contratos são mais eficientes e seguros para os dois lados da transação. Segundo os autores, há outros benefícios advindos do uso de contratos inteligentes com base na tecnologia blockchain, entre eles atualizações em tempo real, precisão, menor risco de manipulação, menos intermediários ou até sua eliminação, custos mais baixos e novos negócios ou modelos operacionais. Para Kehrli (2016), os contratos inteligentes costumam ser o componente central das plataformas blockchain da próxima geração. O artigo explora o que é um contrato inteligente e seu funcionamento, além de enfatizar que a cada semana há um caso de uso do Ethereum.

\section{[QE2] Que tipos de aplicações da tecnologia blockchain estão voltados para a transparência go- vernamental, de acordo com a literatura acadêmica?}

Quanto ao quesito anticorrupção, Kim e Kang (2017) citam que, apesar das oportunidades ao usar a tecnologia blockchain, ela não resolverá todos os problemas, sendo necessário analisar também o lado negativo e as limitações. A conclusão é que essa pode ser uma ferramenta capaz de erradicar fraudes, corrupção e suborno por prover transparência às operações, mas é importante considerar seu efeito cascata negativo, que pode impedir o crescimento sustentável. Dessa forma, é preciso desenvolver uma política bem planejada para maximizar os benefícios do uso da tecnologia blockchain. Adam e Fazekas (2017) destacam que os serviços públicos digitais não são eficazes e que podem até fornecer oportunidades de corrupção. Conforme os autores alegam, se, por um lado, a tecnologia blockchain pode impactar na oferta de informações por oferecer níveis crescentes de transparência e responsabilidade ao setor público ao cortar "intermediários" e reduzir as oportunidades de corrupção, por outro lado, exige mudança de processos, investimentos e treinamentos.

Outra aplicação na área governamental está presente em Young (2018), em que, por meio de contratos inteligentes em blockchain, o relacionamento entre governo e governados pode mudar. Os autores advogam que o próximo passo na evolução para a governança pública é a blockchain, em razão da busca por consenso inerente, escalabilidade e descentralização que o setor começa a exigir. Com esta tecnologia, seria possível controlar as instituições de um governo para criar maior supervisão e impedir que seus atores ajam além do mandato.

A tese de Jalakas (2018), que objetiva a análise da blockchain a partir de uma perspectiva da administração pública, tem a parte empírica concentrada na Estônia, por ser o país líder em termos de adoção da blockchain no setor público. Os autores dizem que o caso da Estônia fornece eficiência operacional, pode aumentar a legitimidade intragovernamental e ser usado para transformar serviços públicos. Uma das ideias propostas é a junção de outros avanços tecnológicos aos da blockchain. O uso de aprendizado de máquina, que pode filtrar os dados para serem mais relevantes, e os recursos dos contratos inteligentes baseados na blockchain, como uma forma segura de automatizar procedimentos organizacionais, podem tornar os serviços públicos personalizados, beneficiando Estado e cidadão.

Hughes et al. (2018) propõem a blockchain para o Reino Unido, cujo objetivo é que os departamentos governamentais façam 1\% de economia em eficiência, adotando a blockchain e outras tecnologias. Para os autores, o Estado deve usar a blockchain para permitir liberdade social, aumentar a eficiência e reconstruir a confiança da sociedade, mas deve-se ter cuidado, pois o Estado não deve ter permissão de usar a tecnologia para invadir a vida dos indivíduos.

Uma área muito criticada pela falta de transparência existente e que sempre passa por incertezas é a votação. No 
artigo de Dogo et al. (2018), é feita uma revisão de artigos acadêmicos sobre a aplicação de tecnologia blockchain para prover segurança na votação eletrônica. Além disso, em países em desenvolvimento, a tecnologia blockchain poderia ser usada como alternativa para sistemas de votação manuais ou digitalizados muito precários.

\section{CONCLUSÕES}

- Como os tomadores de decisão no setor governamental podem ser beneficiados pelos resultados deste trabalho?

O objetivo do artigo foi verificar quais aplicações da tecnologia blockchain estão voltadas para o setor público, contribuindo com os tomadores de decisão no sentido de pensarem na sua inclusão. Esta tecnologia tem ganhado destaque e está crescendo em diversas áreas, como comprovado por esta pesquisa, que encontrou relatos ou possibilidades de uso nos setores elétricos, imobiliários, medicina, gerenciamento de identidades, agricultura, transações comerciais, mercado financeiro, tributação, cidades inteligentes, contratos inteligentes, administração governamental e na votação eletrônica.

Em relação à transparência governamental, a tecnologia blockchain poderia ser útil, trazendo benefícios como a erradicação de fraudes (Kim \& Kang, 2017). Porém, é preciso cautela no uso de serviços públicos digitais, pois eles podem fornecer oportunidades de corrupção (Adam \& Fazekas, 2017). Além disso, deve-se ter cuidado para o Estado não invadir a privacidade dos cidadãos (Hughes et al., 2018). Por isso, governos que priorizam a transparência de seus atos podem se beneficiar com a tecnologia blockchain.

- Quais as conclusões evidenciadas e o que fica como direcionamento futuro de pesquisa relacionado à aplicação do blockchain no setor público?

A RSL realizada indica que a tecnologia em tela tem potencial para ajudar o setor público. Porém, serão necessários futuros levantamento de necessidades para avaliar se a tecnologia blockchain é mais adequada para cada situação proposta. Além disso, em muitas áreas ainda há receio em relação ao uso desta tecnologia, por não haver tantas aplicações em funcionamento, ou seja, por não ter atingido um nível de maturidade desejável. Uma aplicação da blockchain que merece ressalva é na área de votações, pois o voto transparente ou verificável torna-se um problema nos casos em que a votação deve ser secreta, como nos casos de eleições para representantes dos poderes executivo e legislativo.

Como desdobramentos futuros dessa pesquisa, é necessário avaliar que políticas públicas nacionais estão envolvidas com a tecnologia blockchain, diferenciando os casos que são pesquisas ainda em andamento, ou promessa de usos, dos casos em que já é efetivamente empregada. Outro desdobramento seria verificar os diferentes conflitos de interesses em cada um dos cenários, de forma a identificar dificuldades presentes em cada contexto. 


\section{REFERÊNCIAS}

Adam, I., \& Fazekas, M. (2017). Are emerging technologies helping win the fight against corruption in developing countries? In Pathways for prosperity commission background paper series. Oxford: OECD. Recuperado de https://pathwayscommission.bsg.ox.ac.uk/Fight-against -corruption-paper

Barbieri, M., \& Gassen, D. (2017). Blockchain-can this new technology really revolutionize the land registry system? responsible land governance: Towards an evidence based approach. In Proceedings of the annual world bank conference on land and poverty (p. 1-13). Washington, DC. Recuperado de http://www.notaries-of-europe.eu//index.php?pageID= 15101

Barbosa, L. S. (2017). Digital governance for sustainable development. In Conference on e-business, e-services and e-society (p. 88-89). Springer.

Bashir, I. (2017). Mastering blockchain. Packt Publishing.

Benbunan-Fich, R., \& Castellanos, A. (2018). Digitization of land records: From paper to blockchain. In Thirty ninth international conference on information systems.

Berryhill, J., Bourgery, T., \& Hanson, A. (2018). Blockchains unchained: Blockchain technology and its use in the public sector. In Oecd working papers on public governance. doi: $10.1787 / 3 \mathrm{c} 32 \mathrm{c} 429$-en

Dahlquist, O., \& Hagström, L. (2017). Scaling blockchain for the energy sector. Sweden: Uppsala Unviersity. Recuperado de http://uu.diva-portal.org/smash/get/diva2: 1118117/FULLTEXT01.pdf

De La Rosa, J. L., Torres-Padrosa, V., El-Fakdi, A., Gibovic, D., Hornyák, O., Maicher, L., \& Miralles, F. (2017). A survey of blockchain technologies for open innovation. In Proceedings of the 4th annual world open innovation conference. San Francisco, CA. Recuperado de http://eia.udg.edu/ aelfakdi/papers/woic17.pdf

Dogo, E. M., Nwulu, N. I., Olaniyi, O. M., Aigbavboa, C. O., \& Nkonyana, T. (2018). Blockchain 3.0: Towards a secure ballotcoin democracy through a digitized public ledger in developing countries. I-manager's Journal on Digital Signal Processing, 6(2), 24-35. doi: 10.26634/jdp.6.2.15593

Dubey, N. (2018). Roadblocks to application of blockchain in indian agriculture: A study on madhya pradesh (Dissertação de Mestrado, Central European University, School of Public Policy, Budapest, Hungary). Recuperado de http://www .etd.ceu.edu/2018/dubey nikhil.pdf

Duffy, K., Goudovitch, P., \& Fedorov, P. (2016). The application of digital identity in the united states. Recuperado de https://static1.squarespace.com/static/ 59aae5e9a803bb10bedeb03e/t/5a6fd0929140b70f6ceb4849/ 1517277404196/blockchain-digital-identity.pdf

Ferreira, J. E., Pinto, F. G. C., \& Santos, S. C. d. (2017). Estudo de mapeamento sistemático sobre as tendências e desafios do blockchain. Gestão.org, 15, 108-117. doi: 10.21714/1679-18272017v15Ed.p108-117

Figueiredo, V. S., \& Santos, W. J. L. (2014). Transparência e participação social da gestão pública: análise crítica das propostas apresentadas na $\mathrm{i}^{\mathrm{a}}$ conferência nacional sobre transparência pública. Revista Contabilidade e Controladoria, 6(1), 73-88. doi: 10.5380/rcc.v6i1.32082

Flament, C. (2015). Blockchain technology: a general purpose technology for the decentralization of governance? (Tese de Doutorado, ULB Solvay Brussel School, Brussels).
Recuperado de http://dx.doi.org/10.5380/rcc.v6i1.32082

Foroglou, G., \& Tsilidou, A. L. (2015). Further applications of the blockchain. In 12th student conference on managerial science and technology. Recuperado de https://www.researchgate.net/publication/276304843 _Further_applications_of _the_blockchain

Fusco, F., Lunesu, M. I., Pani, F. E., \& Pinna, A. (2018). Crypto-voting, a blockchain based e-voting system. In Proceedings of the 10th international joint conference on knowledge discovery, knowledge engineering and knowledge management (p. 223-227). Seville, Spain. doi: 10.5220/0006962102230227

Gabrich, Y. B., Coelho, I. M., \& Coelho, V. N. (2017). Tendências para sistemas microgrids em cidades inteligentes: Uma visão sobre a blockchain. In Xlix simpósio brasileiro de pesquisa operacional (p. 1-12). Recuperado de http:// www.din.uem.br/ ademir/sbpo/sbpo2017/pdf/169695.pdf

Horiuchi, F. (2008). Bitcoin: A peer-to-peer electronic cash system. Recuperado de https://bitcoin.org/bitcoin.pdf

Horiuchi, F. (2018). Rastreabilidade de um modelo de cadeia produtiva agrícola generalizado em uma rede blockchain [Trabalho de conclusão de curso]. Londrina. Recuperado de http://www.uel.br/cce/dc/wp-content/uploads/ TCC-FELIPE_SEITI_HORIUCHI.pdf

Hughes, E., Graham, L., Rowley, L., \& Lowe, R. (2018). Unlocking blockchain: Embracing new technologies to drive efficiency and empower the citizen. The Journal of the British Blockchain Association, 1(2), 1-15. doi: 10.31585/jbba1-2-(1)2018

Hussein, D. M. E. D. M., Hamed, M., \& Eldeen, N. (2017). A blockchain technology evolution between business process management (bpm) and internet-of-things (iot). nternational Journal of Advanced Computer Science and Applications, 9(8), 442-450. Recuperado de 10.14569/IJACSA.2018.090856

Jalakas, P. (2018). Blockchain from public administration perspective: Case of estonia (Tese de Doutorado, Tallinn University of Technology, Tallinn, Estonia). Recuperado de https://digikogu.taltech.ee/en/Download/d591ed87-3350 -44a1-acb3-f0e184f9dc18

Jardim, J. M. (1995). A face oculta do leviatã: gestão da informação e transparência administrativa. Revista do Serviço Público, 46(1), 137-152. doi: 10.21874/rsp.v59i1.141

Kehrli, J. (2016). Blockchain 2.0-from bitcoin transactions to smart contract applications. Niceideas.ch. Recuperado de https://www.niceideas.ch/roller2/badtrash/entry/ blockchain-2-0-frombitcoin

Khudnev, E. (2017). Blockchain: foundational technology to change the world (Monografia de Graduação, Lapland University of Applied Sciences, School of Business and Culture, Lapand, Finland). Recuperado de https://www.theseus.fi/bitstream/handle/10024/138043/ Evgenii_Khudnev_Thesis.pdf?sequence $=1 \&$ isAllowed $=\mathrm{y}$

Kim, K., \& Kang, T. (2017). Does technology against corruption always lead to benefit? the potential risks and challenges of the blockchain technology. In Oecd's anti-corruption and integrity forum. Recuperado de https://www.oecd.org/cleangovbiz/Integrity-Forum -2017-Kim-Kang-blockchain-technology.pdf

Kussy, M. Y., Pobirchenko, V. V., Shutaieva, E. A., \& Kakutich, Y. Y. (2018). Blockchain technologies as a stimulator of institutional transformations of the world financial system. International Journal of Engineering and 
Technology, 57(3.14), 354-354. Recuperado de https:// www.sciencepubco.com/index.php/ijet/article/view/17025

Lemieux, V. L., Hofman, D., Batista, D., \& Joo, A. (2019). Blockchain technology for recordkeeping. In Arma international educational foundation. Recuperado de http://armaedfoundation.org/wp-content/uploads/ 2019/06/AIEF-Research-Paper-Blockchain-Technology -Recordkeeping.pdf

Lewis, R., McPartland, J., \& Ranjan, R.

(2017). Blockchain and financial market innovation. Economic Perspectives, 7, 1-17. Recuperado de https://www.chicagofed.org/ /media/publications/ economic-perspectives/2017/ep2017-7-pdf.pdf

Livingston, D., Sivaram, V., Freeman, M., \& Fiege, M. (2018). Applying blockchain technology to electric power systems. Recuperado de https://www.jstor.org/stable/pdf/ resrep21340.pdf

Madrigal, A. G. (2016). Transparência, controle de contas públicas e combate à corrupção: controle social na administração pública: a importância da transparência das contas públicas para inibir a corrupção. JusBrasil. Recuperado de https://alexismadrigal.jusbrasil.com.br/artigos/ 446196587/transparencia-controle-de-contas-publicas-e -combate-a-corrupcao

Nasser, Y., Okoye, C., Clark, J., \& Ryan, P. Y. (2018). Blockchains and voting: Somewhere between hype and a panacea. Recuperado de https://users.encs.concordia.ca/ $\sim$ clark/papers/draft_voting.pdf

Pinho, J. A. G. D. (2008). Investigando portais de governo eletrônico de estados no brasil: muita tecnologia, pouca democracia. Revista de Administração Pública, 42(3), 471-493. Recuperado de https://repositorio.ufba.br/ri/bitstream/ri/ 2533/1/v46n1a03.pdf

Prado, O., \& Loureiro, M. R. G. (2006). Governo eletrônico e transparência: avaliação da publicização das contas públicas das capitais brasileiras. Revista Alcance, 13(3), 355-372. doi: 10.14210/alcance.v13n3.p355-372

Ream, J., Chu, Y., \& Schatsky, D. (2016). Upgrading blockchains: Smart contract use cases in industry. deloitte university press. Texas: Deloitte University Press. Recuperado de https://www2.deloitte.com/us/en/insights/focus/signals -for-strategists/using-blockchain-for-smart-contracts.html

Reyes, C. L. (2018). Cryptolaw for distributed ledger technologies: A jurisprudential framework. Jurimetrics, 58, 283-302. Recuperado de https://heinonline.org/HOL/LandingPage?handle=hein .journals $/$ juraba58\&div $=19 \&$ id $=\&$ page $=$

Ruta, M., Scioscia, F., Ieva, S., Capurso, G., Loseto, G., Gramegna, F., ... Di Sciascio, E. (2016). Semantic-enhanced blockchain technology for smart cities and communities. In 3rd italian conference on ict for smart cities $\& 6$ communities. Bari,Italy. Recuperado de http://sisinflab.poliba.it/ publications/2017/RSICLGPD17/

Schatsky, D., \& Muraskin, C. (2015). Beyond bitcoin: Blockchain is coming to disrupt your industry. Deloitte Insights. Recuperado de https://www2.deloitte.com/us/ en/insights/focus/signals-for-strategists/trends-blockchain -bitcoin-security-transparency.html

Singh, N., \& Vardhan, M. (2018). Blockchain based estamp procurement system with efficient consensus mechanism and fast parallel search. Journal of Mechanics of Continua and Mathematical Sciences, 13(4), 73-89. doi: 10.26782/jmcms.2018.10.00007
Staples, M., Chen, S., Falamaki, S., Ponomarev, A., Rimba, P., Tran, A. B., \& Zhu, J. (2017). Risks and opportunities for systems using blockchain and smart contracts. Data61. Recuperado de https://research.csiro.au/data61/wp-content/uploads/ sites/85/2016/08/Blockchain-RisksandOpps-PDF.pdf

Suominen, K. (2018). Closing in on the holy grail of world trade: Using blockchain to expand southeast asia's trade. Geneva, Switzerland: International Centre for Trade and Sustainable Development. Recuperado de https://ictsd.iisd.org/sites/default/files/research/ blockchain_in_southeast_asia_-_suominen.pdf

Tranfield, D., Denyer, D., \& Smart, P. (2003). Towards a methodology for developing evidence informed management knowledge by means of systematic review. British Journal of Management, 14(3), 207-222. doi: 10.1111/14678551.00375

van Nes, W. R. (2017). Don't be fooled by the blocks that it got. blockchain, a revolution or not? (Tese de Doutorado, Erasmus University of Rotterdam, Rotterdam, Netherlands). Recuperado de https://thesis.eur.nl/pub/39530/Nes-WR -van-321311-MA-thesis.pdf

van Rijswijk, L., Hermsen, H., \& Arendsen, R. (2019). Exploring the future of taxation: A blockchain scenario study. In 6th annual tarc workshop. Exeter, UK. Recuperado de https://hdl.handle.net/1887/68772

van Wingerde, M. E. M. (2017). Blockchainenabled self-sovereign identity (Dissertação de Mestrado, Tilburg University, Tilburg, Netherlands). doi: 10.13140/RG.2.2.17693.82406

Veuger, J. (2017). Attention to disruption and blockchain creates a viable real estate economy. Journal of US-China Public Administration, 14(5), 263-285. doi: 10.17265/15486591/2017.05.003

Vishik, C., Karame, G., \& Masucci, D., R. andGoodman. (2017). Blockchain: Perspectives on research, technology and policy. Brussels: Trust in Digital life Association. Recuperado de https://trustindigitallife.eu/wp-content/ uploads/Blockchain-Perspectives-on-Research-Technology -Policy.pdf

Welzel, C. (20017). Blockchain-attack on and chance for the public sector. ERCIM News(110), 24-25. Recuperado de https://ercim-news.ercim.eu/images/stories/ EN110/EN110-web.pdf

Young, S. (2017). Enforcing constitutional rights through computer code. Journal of Law and Technology, 26(1), 5253. Recuperado de https://scholarship.law.edu/jlt/vol26/ iss $1 / 5$

Young, S. (2018). Changing governance models by applying blockchain computing. Economic Perspectives, 26 (2), 53-86. Recuperado de https://scholarship.law.edu/cgi/viewcontent .cgi? article $=1056 \&$ context $=$ jlt

Zhang, P., White, J., Schmidt, D. C., Lenz, G., \& Rosenbloom, S. T. (2018). Fhirchain: applying blockchain to securely and scalably share clinical data. Computational and Structural Biotechnology Journal, 16, 267-278. doi: 10.1016/j.csbj.2018.07.004

Šalehar, D. (2017). Pattern based evaluation of blockchain technology as a catalyst for business model innovation: Exploratory research with focus on the potential implications for e-health. Recuperado de http://resolver.tudelft.nl/uuid: d0afc872-8118-4103-a162-54ecf003677b

Šurda, P. (2012). Economics of bitcoin: is bitcoin an 
alternative to at currencies and gold? (Tese de Doutorado, Wirschafts Universitat Wien, Viena). Recuperado de https://nakamotoinstitute.org/static/docs/economics-of -bitcoin.pdf

Como citar este artigo (APA):

Silva, E. C. C e, \& Marques, R. M. (2021). Blockchain no setor público: uma revisão sistemática de literatura. AtoZ: novas práticas em informação e conhecimento, 10(3), 1 - 11. Recuperado de: http:// dx.doi.org/10.5380/atoz.v10i3.79903 